\title{
La Filaire Dipetalonema dessetae chez Aedes aegypti;
}

\author{
étude du tissu adipeux parasité $(*)$
}

\author{
par G. PETIT et H. SPITALIER-KAVEH
}

Laboratoire de Zoologie (Vers) associé au C.N.R.S., Muséum national d'Histoire naturelle, 43, rue Cuvier, F 75231 Paris Cedex 05

\section{Résumée.}

L'analyse histologique et ultrastructurale du tissu adipeux d'A. aegypti parasité par $D$. dessetae montre que cette Filaire induit localement une synthèse protéique très active (ribosomes, ergatoplasme et appareil de Golgi développés, mitochondries multipliées); cette activation n'est pas sans analogie avec celle provoquée par la $\beta$-ecdysone à la suite d'un repas sanguin. Chez les moustiques qui jeûnent pendant le développement de la Filaire, les réserves (lipides et glycogène) sont vite épuisées, l'ergastoplasme induit présente dès le $7^{\mathrm{e}}$ jour des formes d'involution, le tissu parasité dégénère et le développement de la Filaire est bloqué.

\section{Summary.}

\section{Dipetalonema dessetae - Aedes aegypti: study of the infected adipose tissue.}

Histological and ultrastructural study of the adipose tissue of Aedes aegypti has shown that infection with the filaria Dipetalonema dessetae locally induces a highly active synthesis of proteins (formation of ribosomes, endoplasmic reticulum and Golgi complexes, multiplication of mitochondria). This phenomenon appears similar to the effect of $\beta$-ecdysone after the up-take of blood. Mosquitoes let to starve during the development of the filaria show a quick disappearance of storage substances (lipids and glycogen) and involution forms of the induced ergastoplasma (after seventh days only), so that the infected tissue degenerates and the development of the filaria is arrested.

(*) Ce travail a pu être effectué grâce à une subvention de l'Organisation Mondiale de la Santé.

Accepté le 9 octobre 1978. 


\section{Introduction}

Trois faits nous ont incités à effectuer l'analyse, histologique et ultrastructurale, du tissu adipeux d'Aedes aegypti parasité par Dipetalonema dessetae Bain, 1973.

- La mise en évidence de la nécessité des repas de sang surnuméraires pour obtenir le développement complet de toutes les larves jusqu'au stade infestant (Petit et coll., 1977).

- L'activité physiologique complexe du tissu adipeux : il a une double fonction (stockage de réserves et sécrétion de vitellogénine) et il passe par des phases successives d'activité et de repos, rythmées sur les prises de repas de sang sous le contrôle de la $\beta$-ecdysone; elles s'accompagnent de transformations profondes et réversibles de l'organisation de l'adipocyte (Behan \& Hagedorn, 1978).

- La forte basophilie du tissu adipeux autour des larves de D. dessetae.

\section{Matériel et techniques}

Le porteur de microfilaires est un Proechimys d'élevage (P. oris Thomas, 1904), ayant $1000 \mathrm{mf} / 10 \mu \mathrm{l}$ de sang.

Les expériences se déroulent à $26^{\circ}$; les 2 mues de $D$. dessetae s'effectuent aux $7^{\circ}$ et $13^{\circ}$ jours, et les premières larves infestantes apparaissent au $15^{\circ}$ jour.

Les moustiques, tous émergés le même jour, sont divisés en 3 lots : - un lot prend un repas infestant après l'émergence, puis des repas sur Proechimys sain 3, 6 et 9 jours plus tard ; - un lot-témoin prend uniquement le repas infestant ; — un autre lot-témoin est nourri aux mêmes jours que le premier lot, mais sur Proechimys sain.

Les fixations au Carnoy sont effectuées tous les 3 jours avant les repas et après le $2^{\circ}$ repas, à $1 \mathrm{~h}, 6 \mathrm{~h}$, et $24 \mathrm{~h}$.

Les colorations effectuées sont l'hémalun-éosine pour l'étude topographique, la réaction à l'acide périodique-Schiff contrôlée à l'amylase salivaire pour la détection du glycogène, la réaction d'Unna-Pappenheim contrôlée à la ribonucléase pour la mise en évidence de l'ARN.

C'est avec cette coloration au vert de méthyle-pyronine que les modifications de la taille des noyaux sont analysées; pour éviter des erreurs dues à une hétérogénéité possible du tissu adipeux des moustiques, la région étudiée est toujours la face dorsale des $3^{\circ}$ et $4^{\circ}$ segments ; pour chaque temps de fixation, une quinzaine de noyaux sont dessinés à la chambre claire sur papier millimétré ; la surface de la section de chaque noyau est mesurée en $\mathrm{mm}^{2}$ et la moyenne de ces surfaces, avec l'intervalle de confiance, est indiquée.

Pour la microscopie électronique, les fixations sont effectuées à 3,7 et 11 jours et après le $2^{\circ}$ repas $(6 \mathrm{~h}$ et $24 \mathrm{~h})$; les pièces de corps gras, choisies au niveau des $3^{\circ}$ et $4^{\circ}$ segments abdominaux, sont fixées $1 \mathrm{~h}$ par le glutaraldéhyde à $3 \%$, dans le tampon 
Cacodylate $0,06 \mathrm{M}$ additionné de $0,5 \%$ de $\mathrm{Cacl}_{2}$. Une post-fixation par l'Osmium à $1 \%$ est faite dans un tampon Cacodylate de sodium $0,1 \mathrm{M}$ additionné de $0,5 \%$ de $\mathrm{Cacl}_{2}$. Le mélange araldite-epon est employé comme milieu d'inclusion.

Symboles des légendes des figures

D (appareil de Golgi), G (glycogène), $\mathrm{L}_{1}$ (larve au stade I), $\mathrm{L}_{2}$ (larve au stade II), L (lipides), $\mathrm{M}_{1}$ (Mue I), M (mitochondries), $\mathrm{N}$ (noyau), REG (réticulum endoplasmique granulaire), RL (ribosomes libres), Y (lysosome).

\section{I. - Tissu adipeux des moustiques sains avant et après un repas de sang.}

L'évolution du tissu adipeux après un repas sur Proechimys sain a été analysée afin de pouvoir dissocier clairement les effets du repas de ceux de $D$. dessetae.

Avant le repas (fig. 1 A), les noyaux sont petits, étoilés et sombres, les vacuoles lipidiques sont volumineuses, le glycogène est abondant.

Six heures après le repas, les noyaux sont sphériques, vésiculeux et volumineux (tableau I) ; les travées cytoplasmiques sont épaissies et chargées en ARN, les enclaves lipidiques sont plus petites, le glycogène est en régression ; ces modifications sont encore plus accentuées à $24 \mathrm{~h}$ (fig. $1 \mathrm{~B}$ et $\mathrm{C}$ ).

Tableau I. - Tissu adipeux des Moustiques sains, avant et après le $2^{e}$ repas sur Proechimys sain. Les moyennes des surfaces nucléaires sont calculées chez six Moustiques (deux pour chaque temps de fixation); APS : acide périodique Schiff; REG : reticulum endoplasmique granulaire.

Pour l'ARN cytoplasmique, l'intensité de la réaction a été appréciée en comparant la coloration de l'intestin moyen, toujours très vive $(+++), \dot{a}$ celle du corps gras.

\begin{tabular}{|l|c|c|c|c|}
\hline & \multicolumn{2}{|c|}{ Unna Pappenheim } & APS & Ultrastructure \\
\hline & $\begin{array}{c}\text { ARN } \\
\text { cytoplasmique }\end{array}$ & $\begin{array}{c}\text { Moyenne } \\
\text { des surfaces } \\
\text { nucléaires }\end{array}$ & Glucides & REG \\
\hline Avant le repas .... & +- & $27 \pm 3,5$ & + & - \\
& + & $21,9 \pm 2,8$ & Traces à + & + \\
6 h après le repas & + & $37 \pm 2,3$ & Traces & ++ \\
& ++ & $63,7 \pm 9,1$ & $39,6 \pm 14,2$ & \\
\hline
\end{tabular}

L'analyse ultrastructurale permet de préciser l'évolution de la structure du cytoplasme (fig. 2) : à la $6^{\circ}$ heure, il contient de nombreux ribosomes libres et du reticulum endoplasmique ; à la $24^{\circ}$ heure, presque tout l'ARN cytoplasmique est organisé en ergastoplasme.

48 heures après le repas, ces structures de synthèse ont disparu et l'adipocyte reprend son aspect de cellule au repos (ribosomes rares, traces d'ergastoplasme). 

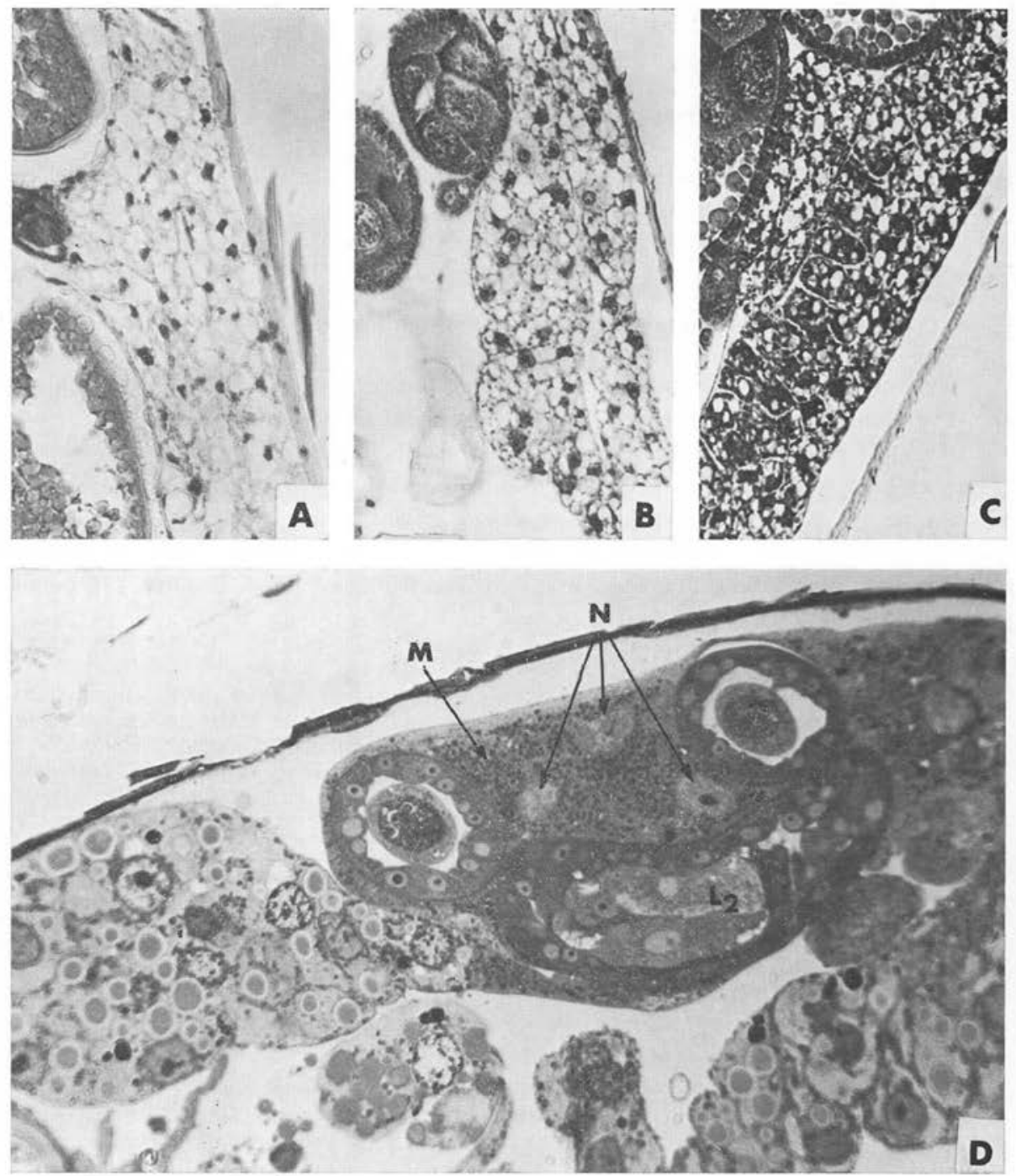

Fig. 1. - Tissu adipeux abdominal d' $A$. aegypti ; A à C, tissu sain; A : juste avant le $2^{\circ}$ repas; $B$ et $C: 6 \mathrm{~h}$ et $24 \mathrm{~h}$ après le $2^{\circ}$ repas de sang; D: tissu parasité, basophile, riche en mitochondries, contenant une larve de 11 jours et tissu sain non modifié. 

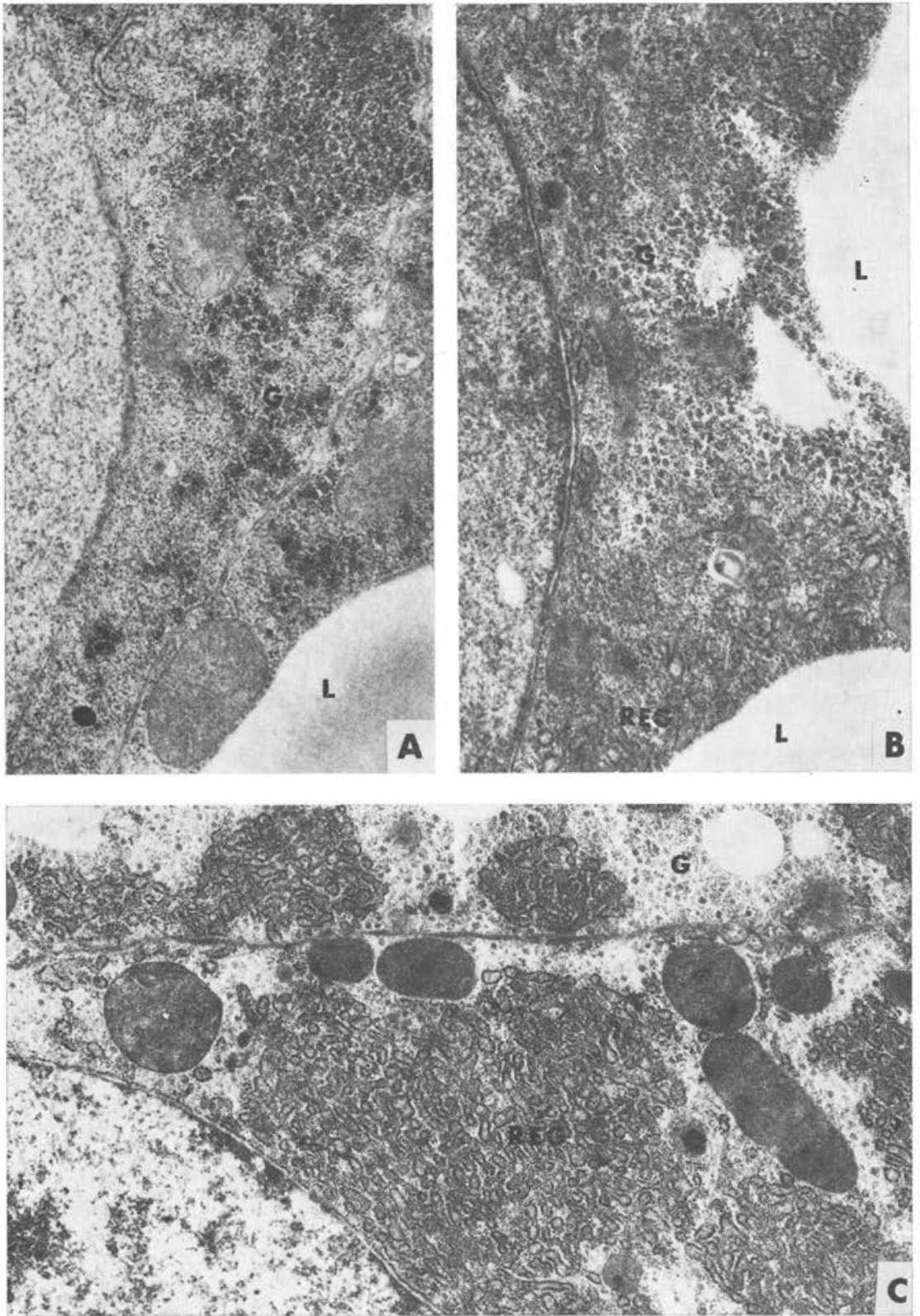

Fig. 2. - Tissu adipeux abdominal d'Aedes aegypti sain au moment du $2^{\circ}$ repas ; A : juste avant le repas (ribosomes, traces d'ergastoplasme, glycogène abondant) $(\times 22.000) ; \mathrm{B}: 6 \mathrm{~h}$ après le repas (ergastoplasme en formation) $(\times 24.000) ; C: 24 \mathrm{~h}$ après le repas (grand développement de l'ergastoplasme) $(\times 20.000)$. 
Nos observations, faites à la suite d'un deuxième repas de sang sur Proechimys, sont conformes à celles de Behan et Hagedorn (1978) faites à la suite d'un premier repas sur Rongeur.

\section{II. - Tissu adipeux parasité.}

1) Résultats de L'analyse histologiQue.

Les larves de $D$. dessetae se développent dans le tissu adipeux abdominal ; toutefois, dans le cas des très fortes infestations, quelques larves (1 ou 2) peuvent être observées dans le tissu adipeux thoracique, très peu volumineux, comparé à celui de l'abdomen.

Le taux de parasitisme est régulièrement plus élevé dans le tissu adipeux dorsal que dans le tissu adipeux ventral, comme le montrent les comptages effectués sur les coupes sagittales de 5 Moustiques, qui ont respectivement 7, 8, 5, 7 et 10 larves dorsales, et $1,2,2,4$ et 3 larves ventrales. Les larves se situent généralement sous le tégument.

A $24 \mathrm{~h}$, la microfilaire s'est installée dans plusieurs cellules adipeuses contiguës et les a désorganisées: les noyaux (environ une dizaine) sont déplacés et regroupés.

Au $3^{\circ}$ jour, avant le premier repas de sang, la larve, généralement disposée en arc de cercle, commence à peine son évolution, mais la région parasitée présente déjà une réaction basophile : les travées cytoplasmiques sont épaissies et basophiles, particulièrement autour des noyaux arrondis, qui sont rassemblés le plus souvent près de la face concave de la larve.

Durant la suite du développement ( 6,9 et 12 jours après le repas infestant), les lipides et le glycogène disparaissent (fig. $1 \mathrm{D})$, la larve est entourée par un cytoplasme riche en ARN, contenant de nombreux granules basophiles. Les noyaux sont arrondis, clairs, à gros nucléole, et généralement plus volumineux que dans les cellules adipeuses non parasitées (tableau II) ; aucune division nucléaire n'est observée.

Tableau II. - Influence de la Filaire sur les noyaux des cellules adipeuses (moustiques nourris).

\begin{tabular}{|l|c|c|}
\hline & \multicolumn{2}{|c|}{ Surface moyenne des noyaux } \\
\hline & Région saine dorsale & Région parasitée dorsale \\
\hline 3 jours, avant le $2^{\mathrm{c}}$ repas ........ & $17,5 \pm 3,8$ & $26,0 \pm 3,7$ \\
& $18,5 \pm 2,2$ & $41,5 \pm 4,4$ \\
3 jours, 6 h après le $2^{\mathrm{e}}$ repas ..... & $44,4 \pm 7,3$ & $61,9 \pm 14,3$ \\
3 jours, 24 h après le $2^{\mathrm{e}}$ repas .... & $34,4 \pm 7,3$ & $41,1 \pm 6,4$ \\
9 jours, avant le $4^{\mathrm{e}}$ repas ........ & $35,5 \pm 5,3$ & $59,3 \pm 8,2$ \\
\hline
\end{tabular}


A 14-15 jours, la larve a complètement envahi le complexe de cellules adipeuses qu'elle parasite. Le cytoplasme a presque totalement disparu. Lors de dissections suivies d'observations à frais, on voit qu'il ne reste plus autour de la larve, redevenue mobile, qu'une sorte de sac évidé, dont la paroi porte, sur la face interne, les noyaux étirés et aplatis des anciens adipocytes.

Aucune différence n'est décelée entre le tissu parasité des moustiques nourris et celui des non nourris.

\section{2) Analyse ultrastructurale.}

Le déplacement des noyaux de la région parasitée paraît bien traduire une véritable transformation de la structure cellulaire initiale en structure syncytiale (fig $1 \mathrm{D}$ et $5 \mathrm{~A}$ ). La basophilie observée dès le $3^{\circ}$ jour correspond à une densité plus grande de ribosomes libres, qui persiste aussi bien chez les moustiques nourris que chez les moustiques non nourris, pendant toute la durée du développement du parasite.

\section{a) Le tissu adipeux parasité et nourri (fig. $3 \mathrm{C}$ et fig. 5).}

$24 \mathrm{~h}$ après le $3^{\mathrm{e}}$ repas de sang $\left(7^{\circ}\right.$ jour), l'ergastoplasme est développé ; il a l'aspect et l'abondance de celui des moustiques sains témoins, mais il présente parfois une organisation concentrique. $48 \mathrm{~h}$ après le $4^{\circ}$ repas (11 jours), les mitochondries sont plus abondantes (1), les dictyosomes remarquablement développés et actifs (émission de vésicules claires ou sombres), l'ergastoplasme persite, mais il est moins abondant qu'au $7^{\circ}$ jour (fig. 5 B).

On observe également quelques lysosomes.

b) Le tissu parasité non nourri (fig. 3 B et fig. 4).

Les particularités observées sur les témoins non nourris sont les suivantes:

- l'ergastoplasme est abondant et il présente des images caractéristiques d'involution (enroulement en couches concentriques, le plus souvent autour de petites enclaves lipidiques, fig. $3 \mathrm{~B}$ et $4 \mathrm{~A}$ et $\mathrm{B})$;

- des fragments de cytoplasme sont isolés par des doubles membranes (fig. 4, D) ;

- des petites vacuoles vides ou à contenu dense, apparaissent et deviennent de plus en plus nombreuses;

- le hyaloplasme se raréfie de plus en plus.

(1) Elles correspondent aux granules basophiles visibles sur les coupes semi-fines (fig. $1 \mathrm{D}$ ). 

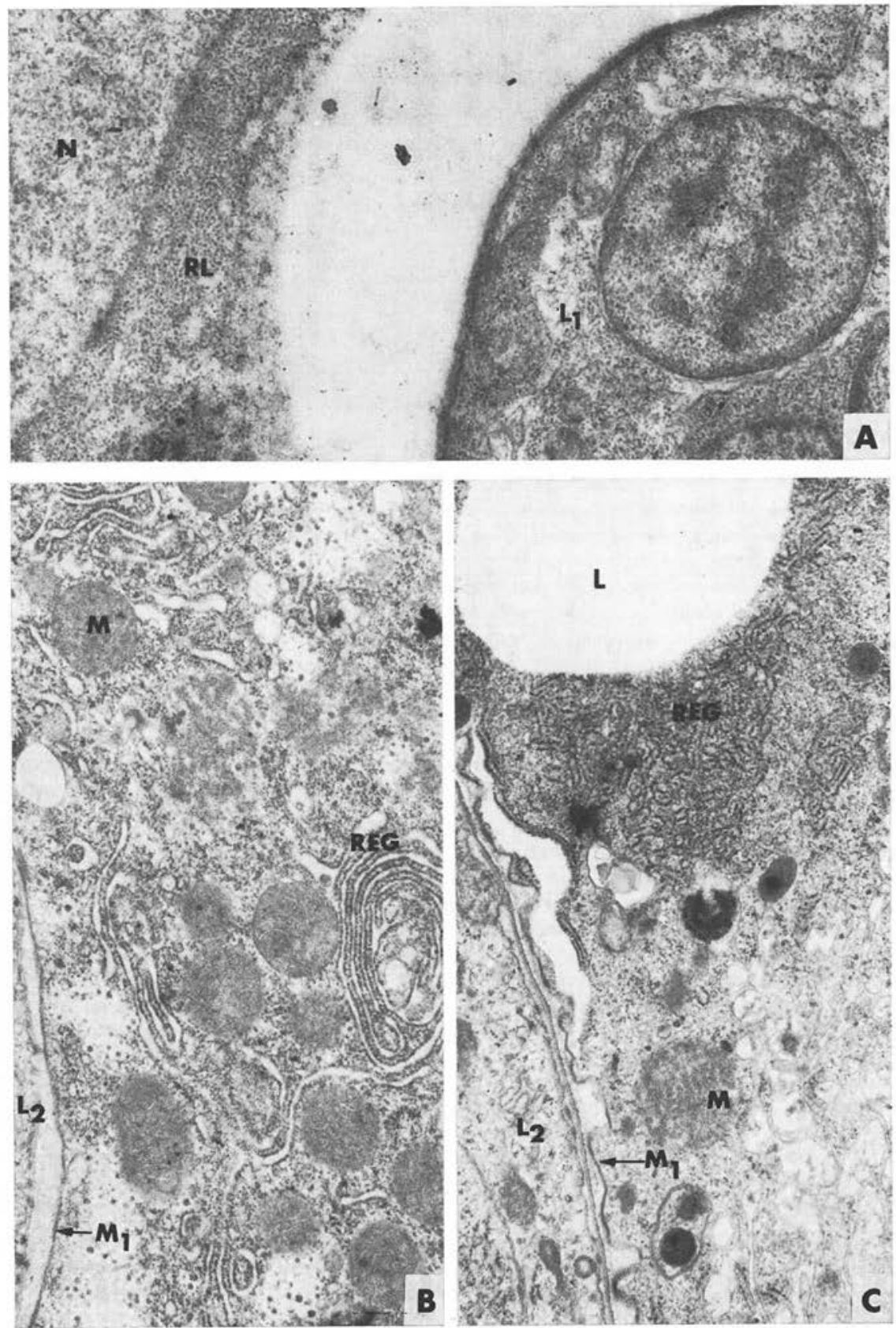

Fig. 3. - Tissu adipeux d'Aedes aegypti parasité $(\times 20.000)$; A: 3 jours après le repas infestant (abondance des ribosomes) ; B : 7 jours, tissu non nourri (ergastoplasme enroulé); $\mathrm{C}: 7$ jours, tissu nourri, $24 \mathrm{~h}$ après le $3^{\mathrm{e}}$ repas de sang (ergastoplasme normal et ribosomes libres, les replis de la membrane basale sont visibles en bas, à droite). 

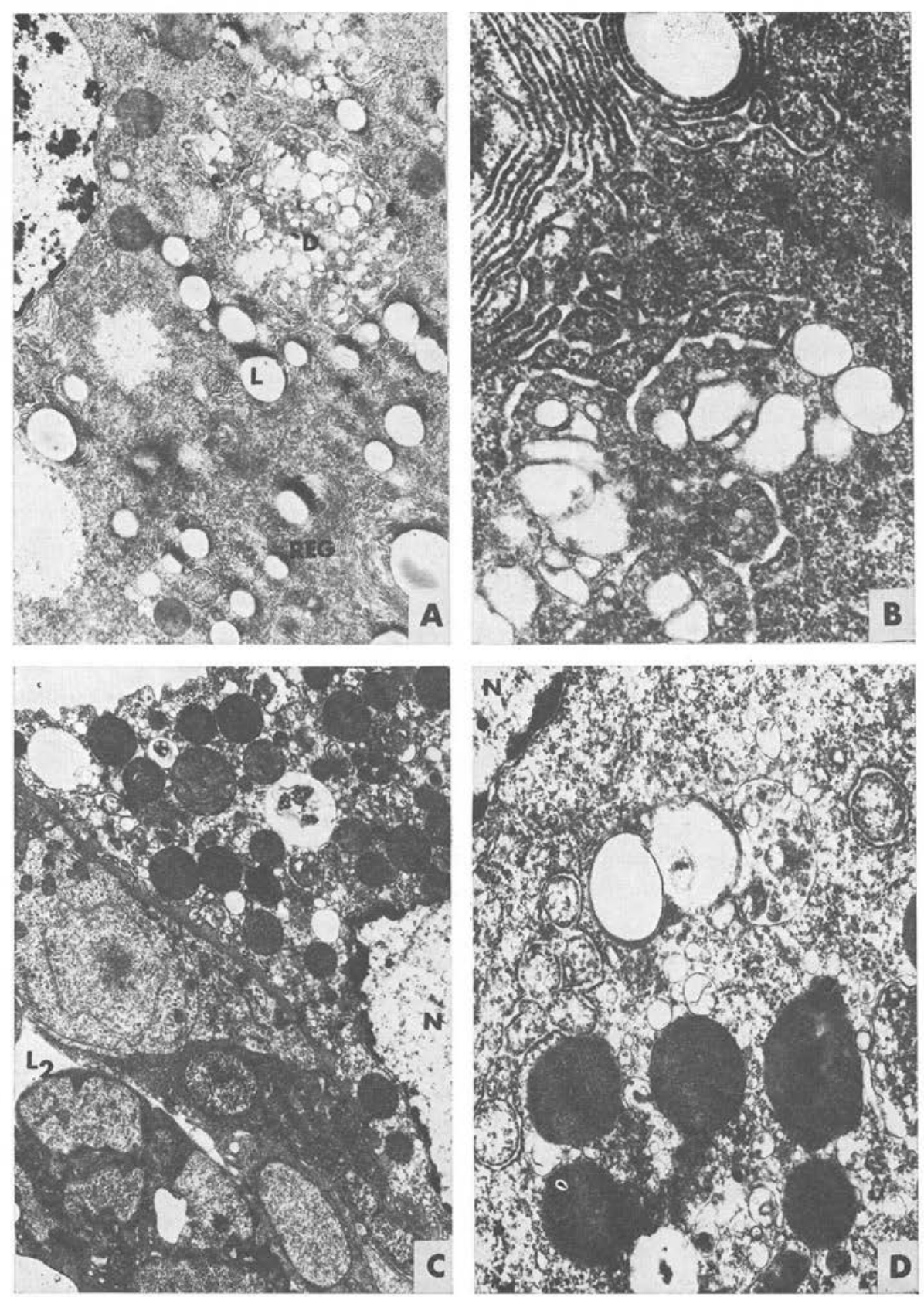

Fig. 4. - Tissu adipeux d'Aedes aegypti parasité et non nourri, au $11^{\circ}$ jour ; A et B : aspect d'une zone parasitée montrant des formes d'involution de l'ergastoplasme et le développement de l'appareil de Golgi (respectivement $\times 13.000$ et $\times 40.000$ ) ; C et D: autre zone parasitée montrant des phénomènes de dégénérescence; vacuoles nombreuses et hyaloplasme raréfié (respectivement $\times 17.000$ et $\times 22.000$ ). 


\section{Discussion}

Nos observations montrent que $D$. dessetae modifie le tissu adipeux qu'elle parasite et que ces transformations sont différentes chez les Moustiques nourris et chez les Moustiques non nourris.

A) Modifications du tissu adipeux par D. dessetae chez les Moustiques nourris.

$D$. dessetae provoque dès son arrivée dans le tissu adipeux la formation d'une structure syncytiale, en regroupant une dizaine de cellules. Elle entraîne également un accroissement du nombre des ribosomes libres et du réticulum endoplasmique ; celui-ci est plus développé entre les repas que dans le tissu sain ; en outre il s'accroît temporairement après chaque repas. Un abondant appareil de Golgi apparaît et les mitochondries se multiplient dans le tissu parasité.

En même temps, les réserves (lipides et glycogène) disparaissent progressivement.

L'ensemble de ces transformations traduit une remarquable activité de synthèse protéique au niveau du tissu parasité, qui est induite par la larve de $D$. dessetae.

B) Différences avec les Moustiques non nourris; interprétation.

L'activation du tissu adipeux est également bien marquée mais le cytoplasme parasité montre dès le $7^{\mathrm{e}}$ jour des images de dégénérescence (réticulum endoplasmique enroulé) qui s'accentuent au $11^{\circ}$ jour (portions de cytoplasme encerclées dans une membrane, nombreuses vacuoles, hyaloplasme raréfié); de telles images sont absentes chez les témoins nourris.

Il semble qu'en l'absence de repas, l'activité de synthèse induite par $D$. dessetae épuise rapidement les réserves du syncytium néoformé puis le syncytium lui-même, qui se lyse. Les larves filariennes ne peuvent plus alors poursuivre leur développement (Petit, sous presse).

C) Comparaison avec d'autres Filaires parasites du tissu adipeux.

Le changement de structure du tissu adipeux parasité paraît être un phénomène général; son «aspect syncytial» a été signalé chez des couples Filaire-vecteur très variés: Loa loa (Guyot, 1778) chez un Chrysops (Lavoipierre, 1958), Dirofilaria roemeri (von Linstow, 1905) chez un Tabanide (Spratt, 1972) et Breinlia booliati Singh et Ho, 1973, chez A. togoi (cf. Ho et coll., 1973). Les images présentées par Schacher et Khalil sur Foleyella philistinae Schacher et Khalil, 1967, chez un Culex suggèrent un phénomène analogue.

Les modifications cytoplasmiques paraissent en général peu perceptibles en histologie classique. Kan et Ho (1972) analysant B. sergenti Mathis et Léger, 1909, chez $A$. aegypti en microscopie électronique, observent le développement d'organites de synthèse (ergastoplasme, dictyosomes...) et concluent à une activation de la synthèse protéique au niveau du tissu adipeux parasité ; leurs images, qui concernent des Moustiques n'ayant eu que le repas infestant, présentent des différences de détail avec celles 

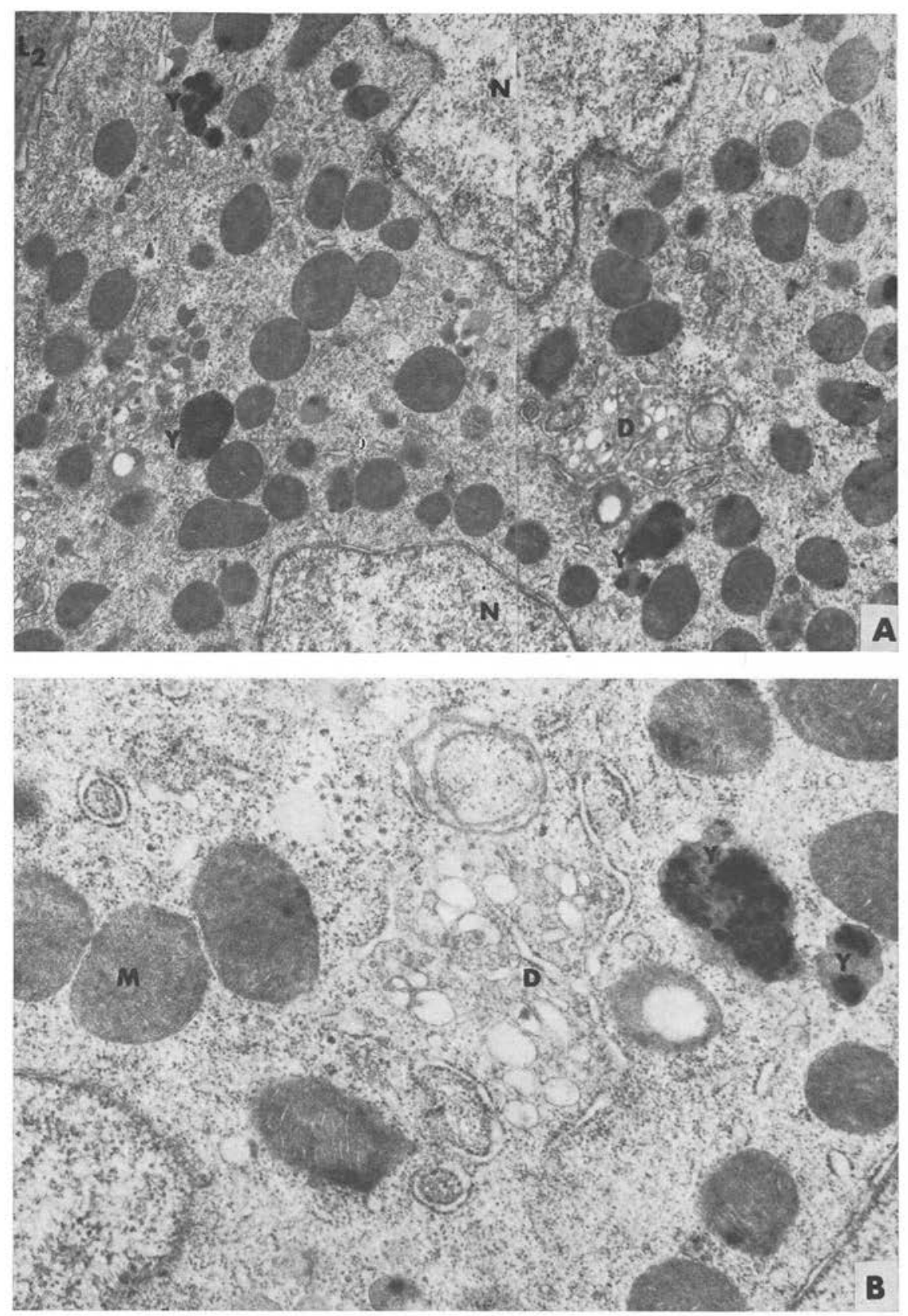

Fig. 5. - Tissu adipeux d'Aedes aegypti parasité et nourri, au $11^{\circ}$ jour (même région que la figure 1, D); A : aspect du tissu parasité montrant la structure syncitiale $(\times 13.000)$; B : détail de cette région parasitée : ergastoplasme, ribosomes, mitochondries et Golgi bien développés $(\times 24.000)$. 
données par $D$. dessetae - $A$. aegypti, et suggèrent que le processus d'activation n'est pas identique chez les deux Filaires.

\section{Conclusions}

Les modifications induites par $D$. dessetae au niveau du tissu adipeux sont profondes (syncitium, activation des structures responsables de la synthèse protéique) et paraissent se rapprocher de celles induites par $B$. sergenti chez le même vecteur.

Le tissu adipeux, dont le rôle normal est d'accumuler les réserves et de synthétiser la vitellogénine, se trouve détourné de ses fonctions par la larve filarienne. Par un stimulus inconnu, mais dont l'effet rappelle celui de la $\beta$-ecdysone, la Filaire active de façon chronique le tissu parasité.

Ce tissu reste toutefois sensible à la $\beta$-ecdysone puisqu'il continue de montrer les variations rythmiques de l'extension de l'ergastoplasme, liées aux repas de sang.

La synthèse des protéines utiles au développement de $D$. dessetae s'effectue, comme celle de la vitellogénine, aux dépens des réserves du tissu adipeux. Ces réserves constituent, par leur volume plus ou moins abondant, un facteur capable de limiter le développement filarien. Chez les Moustiques nourris, l'apport constant de nouveaux métabolites, assure le développement complet de toutes les larves; chez les Moustiques non nourris, l'activité de synthèse entraîne un épuisement des réserves et du syncytium parasité qui se lyse ; le développement des larves est bloqué et le rendement des Moustiques tombe très bas.

\section{Bibliographie}

Behan (M.) et Hagedorn (H.H.), 1978: Ultrastructural changes in the fat body of adult female Aedes aegypti in relationship to vitellogenin synthesis. Cell. Tiss. Res., 186, 499-506.

Kan (S.P.) et Ho (B.C.), 1972: Development of Breinlia sergenti in the fat-body of mosquitoes. II. Ultrastructural changes in the fat-body. J. Med. Entomol., 9, 255-261.

Lavoipierre (M.M.J.), 1958: Studies on the host-parasite relationships of filarial nematodes and their arthropod hosts. I. The sites of development and the migration of Loa loa in Chrysops silacea, the escape of the infective forms from the head of the fly and the effect of the worm on its insect host. Ann. Trop. Med. Parasitol., 52, 103-121.

Petit (G.) : La Filaire Dipetalonema dessetae : phénomènes de régulation et rendement parasitaire chez l'Aedes vecteur. Ann. Parasitol. Hum. Comp. (sous presse).

Petit (G.), Bain (O.) et Spitalier-Kaveh (H.), 1977: Facteurs favorables à la transmission de la Filaire de laboratoire Dipetalonema dessetae. Ann. Parasitol. Hum. Comp., 52, 585-586.

Schacher (J.F.) et Khalil (J.M.), 1968 : Development of Foyella philistinae Schacher and Khalil, 1967 (Nematoda, Filarioidea) in Culex pipiens molestus with notes on pathology in the arthropod. J. Parasitol., 54, 869-878.

Spratt (D.M.), 1972: Natural occurrence, histopathology and developmental stages of Dirofilaria roemeri in the intermediate host. Intern. J. Parasitol., 2, 201-208. 